\title{
XXXIX. On the decay of torsional stress in solutions of gelatine
}

\section{A.O. Rankine B.Sc.}

To cite this article: A.O. Rankine B.Sc. (1906) XXXIX. On the decay of torsional stress in solutions of gelatine, Philosophical Magazine Series 6, 11:64, 447-455, DOI: 10.1080/14786440609463463

To link to this article: http://dx.doi.org/10.1080/14786440609463463

曲 Published online: 16 Apr 2009.

Submit your article to this journal $\sqsubset \pi$

Џll Article views: 2

Q View related articles $\square$

Citing articles: 6 View citing articles 5 
Decay of Torsional Stress in Solutions of Gelatine. 447 and platinum give solutions in water and ethyl malonate in which the particles are negatively charged.

3. The electro-positive, oxidizable metals give solutions in water, methyl alcohol, and ethyl alcohol in which the particles are always positively charged.

4. The velocities of the particles under a known electric force have been determined and the potential differences between the liquid and the particle have been deduced by using the formula

$$
\mathrm{V}=\frac{4 \pi}{\mathrm{K}} \cdot \frac{\mu v}{\mathrm{X}} .
$$

My best thanks are due to Professor J. J. Thomson for his kindly interest and advice during the course of the experiment. I also wish to acknowledge heartily the many helpful suggestions which Mr. W. B. Hardy has kindly given me.

Cavendish Laboratory, Sept. 1905.

XXXIX. On the Decay of Torsional Stress in Solutions of Gelatine. By A. O. RANkIne, B.Se., Assistant in the Physics Department, University College, London *.

THE results of the experiments on the time rate of change 1 of stress in lead wires under constant strain, published in the Phil. Mag. Oct. 1904, suggested an investigation of the laws governing this variation in passing from purely viscous to perfectly elastic bodies. IJead exhibits both elastic and viscous properties to a marked extent, but does not obey the mathematically deduced laws of Maxwell for such bodies. The experimental curve of stress against time when the stress remains constant is not of the form $\mathrm{W}=\mathrm{W}_{0} e^{-\lambda t}$, but is represented fairly accurately by the equation

$$
\mathrm{W}=\mathrm{W}_{0}-a \log (p t+1) .
$$

The question arises, "Does a similar law hold when the viscous and elastic properties are combined in proportions differing from those in lead; and, if not, how does the law change in passing from the perfectly elastic to the purely viscous cases?" In order to find an answer experimentally, one must be provided with a series of substances of the same kind possessing elastic and viscous properties combined in different proportions and extending from the purely viscous on the one side to the perfectly elastic on the other. Now solutions of gelatine in water fulfil these conditions. Solutions of very low concentration possess no elastic properties,

Communicated by Prof. F. T. Trouton, F.R.S 
and at the same time it is possible with high concentrations to make specimens which are perfectly elastic for fairly large stresses. Intermediate concentrations give elastic and viscous properties in varied proportions. Gelatine was therefore chosen for the investigation of the question proposed above.

The consistency of solutions of gelatine in water which have been allowed to congeal is very varied in character. All of these, however, seem to possess, to a greater or less degree, elastic properties. It also appears that any particular specimen has a definite elastic limit provided that none of the conditions which determine its consistency vary. It may be subjected to a stress of less than a certain limiting value, and the resulting strain will be constant with regard to time and it will recover on the removal of the stress to its original state. If, however, this limiting stress is exceeded, the strain is no longer constant but increases with time. The removal of the stress does not now produce complete recovery, although it should be noted that if the stress is only applied momentarily the recovery is very nearly complete.

Now suppose a specimen of jelly stressed beyond its elastic limit. There will result an immediate strain followed by a gradual increase with time. This increase, however, can be prevented by the gradual removal of the stress, and in order to keep the strain constant this removal must be continued until the remaining stress is equal to the elastic limit of stress for the particular specimen.

It was to investigate this change in the special case of the rate of decrease of couple in solutions of gelatine twisted through a constant amount, that the following experiments were carried out.

\section{Method of Experiment.}

The method used has been to apply equal torsional stresses to gelatine solutions of different concentrations, to maintain the strain always equal to the initial immediate strain, and to record the rate of falling off of the stress. It was arranged that the removal of the stress should be effected automatically by a method very similar to that used by Professor Trouton and myself in our experiments on lead wires (Phil. Mag. October 1904).

The interspace between two concentric glass cylinders is filled with a prepared solution of gelatine which is allowed to set. A torsional stress can then be produced in the jelly by twisting the inner cylinder and retaining the outer one fixed. This inner cylinder forms the lower part A B of tigure 1, and is rigidly fixed to the brass.piece $C D$ which is itself supported 
vertically by means of two steel points at $\mathrm{C}$ and $\mathrm{E}$. The brass projection $F \mathrm{G}$ is perpendicular to the direction of the cylinder and has a platiuum point at its end $G$ which, when the cylinder is twisted by means of threads passing round the wheel $\mathrm{H}$, can be brought into contact with a fixed platinum plate, thus completing an electrical circuit through a PostOffice relay. The whole is supported on a stand (figure 2 ). The threads producing the couple pass over two pulleys $\mathrm{K} \mathrm{K}$ and are attached to one arm of a balance. From this same arm hangs a tin vessel $L$, containing water with a layer of oil on the top to prevent evaporation. The weight of this vessel and a certain amount of the water it contains is counterbalanced by weights placed on the other pan of the balance so. that the tension in the threads at any time is

Fig. 1. equal to the difference between the two weights. VC If necessary, therefore, all the tension may be removed by drawing off sufficient water from the vessel L. The water is run off by means of a siphon through the electrically controlled valve $M$ into a second vessel $N$, which is suspended from a spring, and the weight of water in the vessel $\mathrm{N}$ at any time is recorded on the revolving drum $\mathrm{O}$ by the pen $\mathrm{P}$.

The current passing through the platinum point and plate before mentioned is far too small to itself actuate the water valve $M$, and it is necessary to introduce two relays $R_{1}$ and $\mathrm{R}_{2}$ to effect a sufficient increase of current.

Contact between the platinum point and plate causes the water valve to open and a small quantity of water leares L. This, however, reduces the torsional stress in the jelly, a small recovery takes place, contact is broken and the valve closes. By means of this intermittent action the inner glass cylinder which is imbedded in the gelatine is always kept in the same position, $i$.e. the strain remains constant; and the curve drawn on the revolving drum gives the weight removed at any time, and since this weight is proportional to the torsional stress its. value may be taken as a measure of the latter.

Dimensions :- The total length of the outer cylinder was about $50 \mathrm{cms}$., and the end of the inner cylinder was about $20 \mathrm{cms}$. from the bottom of the former. In all cases the length of the inner cylinder imbedded in jelly was the same, viz. about $26 \mathrm{cms}$. The diameter of 
the outer cylinder was about $3 \mathrm{cms}$. and that of the inner $1 \mathrm{~cm}$. The diameter of the wheel about which the couple was applied was about $4.5 \mathrm{cms}$.

Experiments were made with $3 \cdot 4,4,4 \cdot 2,4 \cdot 4$, and 4.5 per cent. solutions, and in each case the couple was produced by $500 \mathrm{gr}$. wt. acting round the wheel $\mathrm{H}$. The platinum plate was then adjusted so as to come into contact with the point $G$, and the action of the valve began.

Fig. 2.

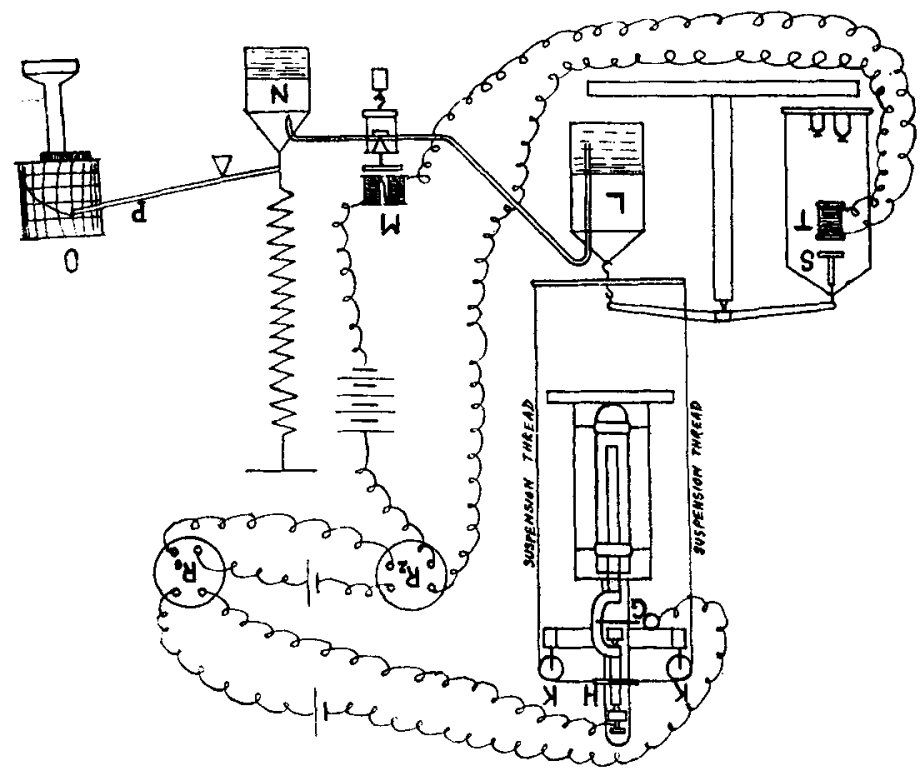

The friction of the points $\mathrm{C}, \mathrm{E}$ and the pulleys $\mathrm{K}, \mathrm{K}$ was not large, being only equivalent to a couple produced by $5 \mathrm{gr}$. wt. acting round the wheel $\mathrm{H}$, but in order to obtain a smooth curve it was necessary to eliminate it. If it is not allowed for, the pen draws a curve consisting of a series of alternate vertical and horizontal lines, each upward step being equivalent to the frictional couple. This difficulty was overcome in the following way :-An iron plate $S$ (fig. 2) suspended from the second arm of the balance is adjusted to such a distance from the electromagnet $T$ that when a given current flows in $T, S$ is pulled downwards with a force equal to the frictional force. This causes the tension in the strings to be relieved to that extent. The current flows in $T$ whenever the water valve opens. Hence the platinum 
point is free to recover without baving first to overcome friction. In this way a series of smooth curves was obtained.

Another precaution had to be taken. Preliminary experiments sufficed to show that the consistency of any specimen of jelly depended largely on the temperature. For purposes of comparison between solutions of different concentrations, therefore, it was necessary to maintain them throughout the experiment as far as possible at equal temperatures. To effect this the outer cylinder containing the jelly was surrounded by a water-jacket which was itself wrapped round and round with felt. It was then possible to keep the temperature of the jelly fairly constant for about three hours.

The following table shows the variation of temperature in the actual experiments :-

\begin{tabular}{|r|c|c|c|}
\hline Experiment. & $\begin{array}{c}\text { Percentage } \\
\text { of Gelatine. }\end{array}$ & $\begin{array}{c}\text { Maximum \& Minimum } \\
\text { Temperatures. } \\
\text { Centigrade degrees. }\end{array}$ & $\begin{array}{c}\text { Mean Temperature } \\
\text { for two hours. }\end{array}$ \\
\hline $1 \ldots \ldots \ldots$. & $3 \cdot 4$ & $\mathrm{~J} \cdot 32-16 \cdot 44$ & $16 \cdot 38$ \\
$2 \ldots \ldots \ldots$. & $4 \cdot 0$ & $16 \cdot 23-16 \cdot 59$ & $16 \cdot 41$ \\
$3 \ldots \ldots \ldots$. & 4.5 & $16 \cdot 10-16 \cdot 52$ & $16 \cdot 31$ \\
$4 \ldots \ldots \ldots$. & $4 \cdot 2$ & $16 \cdot 15-16 \cdot 63$ & $16 \cdot 39$ \\
$5 \ldots \ldots \ldots$. & $4 \cdot 4$ & $16 \cdot 18-16 \cdot 76$ & $16 \cdot 47$ \\
\hline
\end{tabular}

II. Preparation of Solutions.

The consistency of any specimen of jeliy does not solely depend on the percentage of gelatine present and the temperature. It depends also on the way in which the solution is prepared and the time allowed for setting. Thus, if one solution of gelatine be boiled for some time and another of equal concentration be made simply by dissolving the gelatine in hot water without boiling, then, even if the times of setting are the same, the two have different consistencies. A gradual hardening, too, occurs in any jelly even after the temperature of the atmosphere has been reached.

In order to compare specimens varying only in concentration, it was essential to ensure that all the other conditions determining the consistency remained constant.

The temperature was kept constant in the way before described. All the solutions were prepared by simply dissolving the gelatine in water that had just been boiled and making up to the required concentration by the addition of hot water. The liquid (equal volumes in each case) was then poured into the interspace between the two glass cylinders and

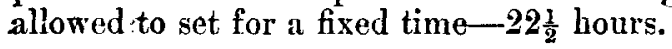




\section{Results.}

The curves shown in figure 3 are copied from the experimental curves. The break in the curve, which probably indicates the point at which the elastic limit is reached, will

Fig. 3.

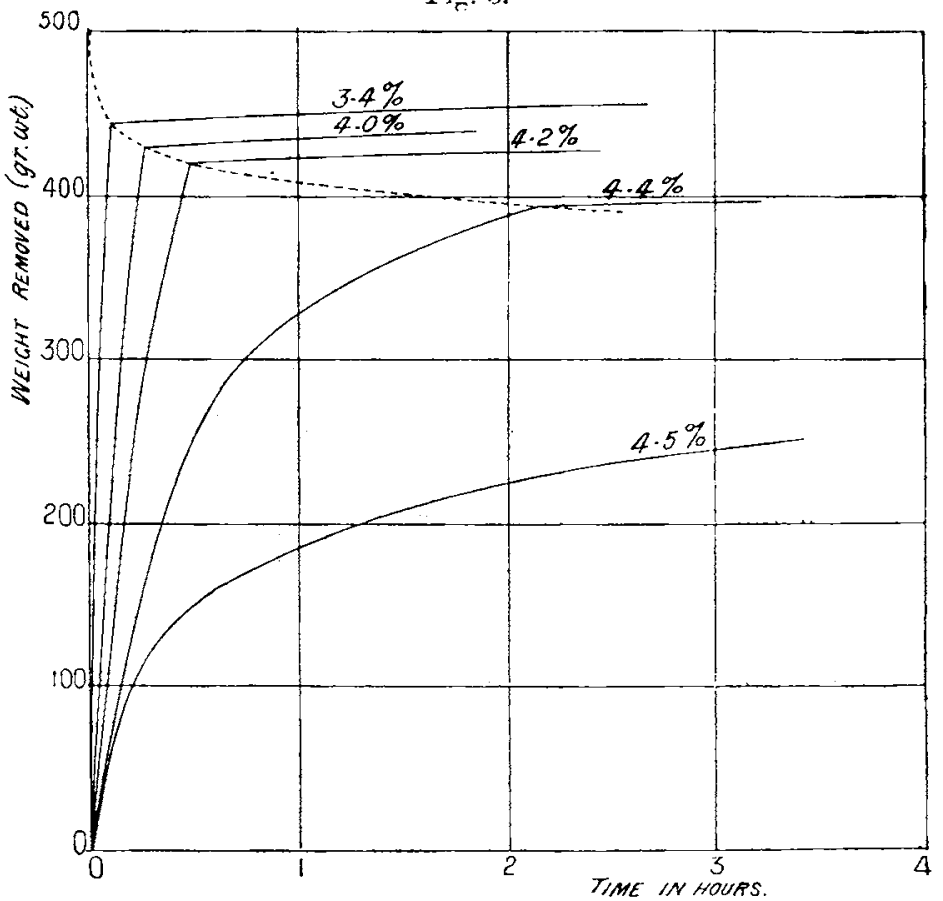

be noticed in the cases of the $3 \cdot 4,4 \cdot 0,4 \cdot 2$, and $4 \cdot 4$ per cent. specimens. It was not found practicable to maintain the temperature constant for a sufficient time to obtain such a break in the 4.5 per cent. curve. It will be seen, too, that these points lie on a curve $\mathrm{which}$, from theoretical considerations, must pass through the point $t=0 \mathrm{~W}=500$. For in the limiting case when the substance possesses no elasticity, it follows that it has no tendency to recover when stress is removed; and hence the whole weight would be removed instantaneously, and the curve representing the removal of weight with time would simply consist of the two straight lines at right angles, $t=0$ and $W=500$. If we suppose that all the curves do break when they meet this dotted curve, then it is obvious from the diagram that the breaking point of the $4 \cdot \bar{\partial}$ per cent. curve must be at a considerably greater time and a considerably less weight than that of the $4 \cdot 4$ per cent. curve. This supposition is to some extent justified by 
the curve shown in figure 4, which represents final stress against concentration. This curve in the region 4.4 per cent. concentration is very steep, and a small change of concentration makes a comparatively large change in the elastic limit.

Returning now to figure 3, it will be seen that the curves are not quite horizontal after the break has been reached; that is to say, the elasticity still gradually diminishes. Possibly this may be accounted for by the fact that during each experiment the temperature was slowly rising, which condition would tend to decrease the elastic limit of the specimen.

No type of equation has been found which will fit all the curves, but an equation of the form $\mathrm{W}=a \log (p t+1)$ fits the $4 \cdot 5$ per cent. curve with considerable exactness This particular curve is of the same type as those for lead wire treated in a similar manner ("On the stretching and torsion of lead wire beyond its elastic limit," Phil. Mag. Oct. 1904), for not only do equations of the same form approximately represent the curves, but both curves show similar departures from those equations. The above equation also satisfactorily represents all curves corresponding to higher concentrations than 4.5 per cent.; these are not shown in the diagram.

It is noticeable also that the dotted curve passing through the points of breakage of the four upper curves is of like

Fig. 4.

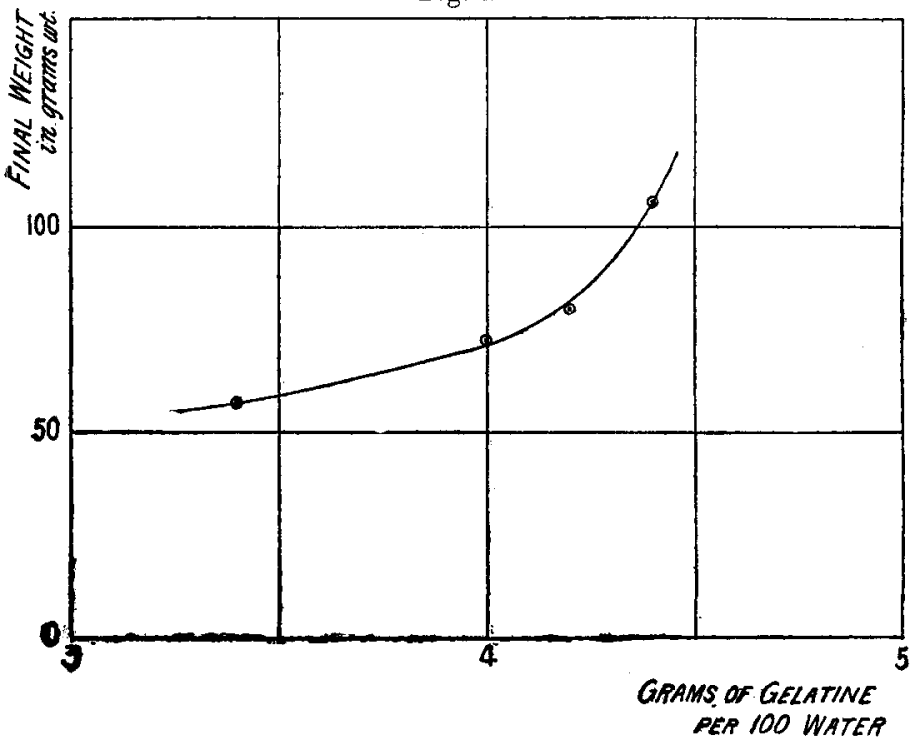

shape, being fairly represented by an equation of the form $\mathrm{W}=\mathrm{W}_{0}-a \log (p t+1)$.

Figure 4 shows the final weight, which is of course 


\section{Decay of Torsional Stress in Solutions of Gelatine.}

proportional to the couple, plotted against concentration, and, if we regard each specimen as being elastic to the extent shown, it indicates that as the concentration increases the elastic limit increases more and more rapidly.

The elastic limits and the times at which they are reached are shown in the following table:-

\begin{tabular}{|c|c|c|}
\hline Concentration. & Elastic Limit. & $\begin{array}{c}\text { Time of reaching } \\
\text { limit. }\end{array}$ \\
\hline $3 \cdot 4$ & $57 \mathrm{gr}$. wt. & 0.114 hour. \\
\hline $4 \cdot 0$ & $72 \quad$ & 0.274 \\
\hline $4 \cdot 2$ & $80 \quad$, & $0.496 \quad$, \\
\hline $4 \cdot 4$ & 106, & $2 \cdot 160$ hours. \\
\hline
\end{tabular}

\section{Conclusions.}

The following conclusions may be drawn from the results of these experiments :-

1. That any specimen, the concentration of which exceeds some fixed limit, is capable of permanently supporting a certain stress.

2 . That the nature of the variation of stress for constant strain depends on the amount by which the limiting stress is exceeded. Thus we see that the curves of the $3 \cdot 4,4 \cdot 0$, and $4 \cdot 2$ per cent. concentrations differ in kind from that representing the 4.5 per cent. concentration; that is to say, they are not represented by equations of the type $\mathrm{W}=a \log (p t+1)$. Owing to the smallness of the time scale it is impossible to reasonably fit equations to them, and it is intended to repeat these determinations using a more quickly moving drum, in order to obtain a more open scale. But the $4 \cdot 4$ per cent. curve is not of the same type as the 4.5 per cent. No equation of the form suitable to the latter fits it. It appears to partake of the shape of both upper and lower curves. $W_{\theta}$ conclude, therefore, that for specimens of greater concentration, i.e. those in which the elastic preponderates over the viscous, the variation of stress under constant strain follows the logarithm law $\mathrm{W}=\mathrm{W}_{0}-a \log (p t+1)$ just as in the case of lead; on the other hand, if the concentration is low and viscosity is the more prominent property, some other law is followed, and solutions of intermediate strength are governed by a combination of these laws.

3. That, in some cases at least, the whole of the excess 
The $H$ Theorem and the Dynamical Theory of Gases. 455 stress is dissipated within a finite time, contrary to what we should expect from theoretical considerations. At any rate, whether or not it is admitted that the breaks in the curves indicate that the elastic limit has been reached, it is certain that at these points a discontinuity occurs, the law on the one side being entirely different from that on the other side.

In conclusion I wish to thank Professor Trouton and Professor Porter for the kind interest they have shown in the work, and for the suggestions which have enabled me to surmount many of the difficulties which have arisen during the investigation.

XL. The H Theorem and Professor J. H. Jeans's Dynamical Theory of Gases. By S. H. Burbury, F.R.S.*

1. ROLTZMANN'S H Theorem professes to prove that whenever the state of a gas is other than the normal state, $\frac{d \mathrm{H}}{d t}$ is negative, and $\mathrm{H}$ is a minimum in the normal state. $\mathrm{H}$ is the function $\int_{-\infty}^{+\infty}(f \log f-f) d u d v d v ; f d u d v d v$ being: the number per unit of volume of molecules whose component velocities lie between $u$ and $u+d u, v$ and $v+d v$, $w$ and $u+d w$. In the normal state as usually understood, $f=\mathrm{A} e^{-h n\left(u^{2}+v^{2}+r^{2}\right)}$. As a consequence of the theorem, $\mathrm{H}$, once minimum, remains minimum for ever, making the process irreversible. Then the objection was made that the Theorem, proving $\frac{d \mathrm{H}}{d t}$ to be necessarily negative, if not zero, proves too much, because we have only to reverse all the velocities simultaneously, and the system will retrace its course with $\mathrm{H}$ increasing. The question is how to explain the paradox.

2. Professor J. H. Jeans, a very strong mathematician, has dealt with this question in his recent work on the Dynamical Theory of Gases. He gives (art.11) a definition of the density, $v$, of a gas at a point $\mathrm{P}$ : namely, $v$ is the number of molecules in a volume at $P$, which volume is very great compared with the mean molecular distance, but very small compared with the scale of variation of density of the gas. The definition is free from ambiguity. But it ignores variations of density on the scale of the intermolecular distances.

* Communicated by the Author. 